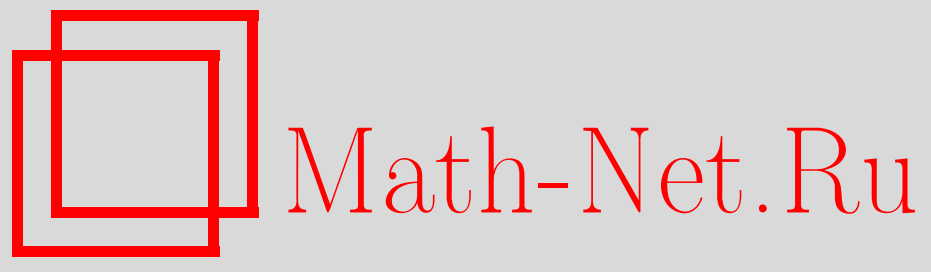

А. В. Комлов, С. П. Суетин, Асимптотическая формула для двухточечного аналога полиномов Якоби, УМH, 2013, том 68, выпуск 4, 183-184

DOI: https://doi.org/10.4213/rm9545

Использование Общероссийского математического портала Math-Net.Ru подразумевает, что вы прочитали и согласны с пользовательским соглашением http://www . mathnet.ru/rus/agreement

Параметры загрузки:

IP: 54.147 .182 .235

26 апреля 2023 г., 12:02:26

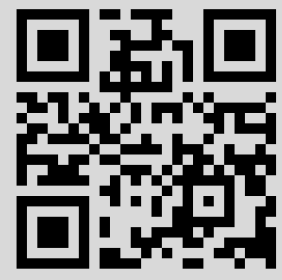




\section{Асимптотическая формула для двухточечного аналога полиномов Якоби}

\section{А. В. Комлов, С. П. Суетин}

1. В работе В. И. Буслаева [3] в классе многозначных аналитических функций с конечным числом точек ветвления получено обобщение классической теоремы Г. Шталя [9] на случай двухточечных (в точках $z=0$ и $z=\infty$ ) аппроксимаций Паде (АП). Принципиальное отличие теоремы Буслаева состоит в том, что соответствующий $\mathscr{S}$-компакт $\Gamma$ в типичном случае делит риманову сферу $\overline{\mathbb{C}}$ на две области $D_{0} \ni 0$ и $D_{\infty} \ni \infty$ (ср. [5]). В [3], в частности, доказано, что существует предельное распределение нулей двухточечных полиномов Паде, совпадающее с равновесной мерой компакта Г. Тем самым в [3] решен вопрос об асимптотическом поведении "почти всех" нулей таких полиномов Паде за исключением $o(n)$ из них. Вопрос о полном описании асимптотического поведения всех нулей двухточечных полиномов Паде остается открытым (даже для классических АП эта задача до сих пор полностью не решена, см. [10], [2]). В настоящей работе эта задача решается для двухточечных АП функций следующего вида:

$$
f(z)=\left(z-a_{1}\right)^{\alpha}\left(z-a_{2}\right)^{-\alpha}, \quad \text { где } \alpha \in \mathbb{C} \backslash \mathbb{Q},
$$

$a_{1}, a_{2}$ - две различные точки в $\mathbb{C} \backslash\{0\}$. Для обычных АП общий случай сводится к случаю $a_{1}=1, a_{2}=-1$ и полиномы Паде - это полиномы Якоби $P_{n}^{(\alpha,-\alpha)}(z)$, для которых справедлива асимптотическая формула [11; гл. 8, формула (8.21.9)]

$$
P_{n}^{(\alpha,-\alpha)}(z)=\left(\frac{z+1}{z-1}\right)^{\alpha / 2} \frac{1}{\left(z^{2}-1\right)^{1 / 4}}\left(z+\left(z^{2}-1\right)^{1 / 2}\right)^{n+1 / 2}, \quad z \notin[-1,1] .
$$

Для функций вида (1) соответствующие двухточечные полиномы Паде $Q_{n}(z)$ (cм. (3)) естественно рассматривать как двухточечный аналог полиномов Якоби (ср. [7]). Мы предполагаем в дальнейшем, что точки $a_{1}, a_{2}$ удовлетворяют условиям $\left|a_{1}\right|<1<\left|a_{2}\right|$, $a_{1} a_{2}=1, a_{1}, a_{2} \notin \mathbb{R}, i \mathbb{R}$.

Пусть $f_{0}$ - элемент функции $f$, голоморфный в точке $z=0, f_{\infty}$ - элемент, голоморфный в точке $z=\infty$, а полиномы $P_{n}, Q_{n} \in \mathbb{C}_{n}[z], Q_{n} \not \equiv 0$, таковы, что

$$
R_{n}(z):=\left(Q_{n} f-P_{n}\right)(z)= \begin{cases}O\left(z^{n+1}\right), & z \rightarrow 0, \\ O(1), & z \rightarrow \infty .\end{cases}
$$

В (3) в окрестности точки $z=0$ берется ветвь $f_{0}$ функции $f$, в окрестности точки $z=\infty$ - ветвь $f_{\infty}$. Полиномы $P_{n}, Q_{n}$ определены неоднозначно, но рациональная функция $P_{n} / Q_{n}$ единственна и называется двухточечной аппроксимацией Паде функции $f$ (см. [1], [3]). Здесь рассматривается только тот случай, когда для получения элемента $f_{\infty}$ аналитическим продолжением элемента $f_{0}$ необходимо по крайней мере один раз обойти точку ветвления $a_{1}$ или $a_{2}$. Известно [4], [3], что в этом случае компакт Буслаева $\Gamma=\Gamma_{\text {Вus }}\left(a_{1}, a_{2} ; f_{0}, f_{\infty}\right)$ разбивает $\overline{\mathbb{C}}$ на две области $D_{0} \ni 0$ и $D_{\infty} \ni \infty$ и допускает следующее описание. Компакт $\Gamma$ состоит из замыканий критических траекторий квадратичного дифференциала $-V(z) /\left(A(z) z^{2}\right) d z^{2}$, где $A(z)=\left(z-a_{1}\right)\left(z-a_{2}\right), V(z)=\left(z-v_{1}\right)\left(z-v_{2}\right),\left|v_{1}\right|<1<\left|v_{2}\right|, v_{1} v_{2}=1 ; v_{1}, v_{2}$ - точки Чеботарёва для $\Gamma$.

Пусть выполнены следующие условия "общего положения" на расположение точек $a_{1}, a_{2}$ и величину показателя $\alpha: \operatorname{deg} P_{n}=\operatorname{deg} Q_{n}=n, R_{n}(z)=c_{1} z^{n+1}+\cdots$, $c_{1} \neq 0, z \rightarrow 0, R_{n}(z)=c_{2}+c / z+\cdots, c_{2} \neq 0, z \rightarrow \infty$.

Работа выполнена при поддержке РФФИ (гранты № 11-01-00330-а, 12-01-31284-мол-а, 12-01-33020-мол-а-вед, 13-01-12430-офи-м2, 13-01-00622-а) и программы "Ведущие научные школы" (грант НШ-4664.2012.1).

DOI: $10.4213 / \mathrm{rm} 9545$ 
2. Положим

$$
\psi(z)=\frac{1}{2} \int_{a_{1}}^{z} \frac{1}{t} \sqrt{\frac{V}{A}(t)} d t, \quad z \in D_{0},
$$

где путь интегрирования лежит в $D_{0}$ и выбрана такая ветвь корня, что $\sqrt{1}=-1$. Пусть $\mathfrak{R}_{2}$ - двулистная эллиптическая риманова поверхность, заданная уравнением $W^{2}=A(z) V(z), d \Omega_{1}(z)=(A(z) V(z))^{-1 / 2} d z$ - абелев дифференциал первого рода на $\mathfrak{R}_{2}, z_{n}$ - решение уравнения

$$
\pm \int_{a_{1}}^{z_{n}} d \Omega_{1}=2 n \int_{0}^{\infty} d \Omega_{1}+\rho \quad\left(\bmod \text { periods }\left(d \Omega_{1}\right)\right),
$$

где $\rho$ не зависит от $n$, знак “土” соответствует тому, на каком из двух листов $\mathfrak{R}_{2}$ лежит точка $z_{n}$; решение уравнения (5) существует и единственно. Положим

$$
\Omega(z ; \zeta)=\zeta \sqrt{\frac{A}{V}(\zeta)} \int_{a_{1}}^{z} \frac{1}{t} \sqrt{\frac{V}{A}(t)} \frac{d t}{t-\zeta}, \quad u_{j}(z)=\int_{a_{1}}^{z} \frac{1}{t} \sqrt{\frac{V}{A}(t)} \frac{d t}{t-v_{j}}, \quad j=1,2,
$$

$u(z ; \zeta)=I_{1}(\zeta) u_{1}(z)+I_{2}(\zeta) u_{2}(z)$, где $I_{1}, I_{2}$ - некоторые явно вычисляемые эллиптические интегралы; путь интегрирования в (4) и (6) выбирается один и тот же.

Справедлива следующая

Теорема 1. Пусть $z_{n}$ - решение уравнения (5) с определенным $\rho=\rho\left(f_{0}, f_{\infty}\right) u$ для некоторой подпоследовательности $\Lambda$ выполняется условие (ср. [6; (3.3)]):

$$
0<C_{1} \leqslant\left|z_{n}\right| \leqslant C_{2}<\infty, \quad\left|A\left(z_{n}\right) V\left(z_{n}\right)\right| \geqslant C_{3}>0, \quad n \in \Lambda .
$$

Тогда в $D_{0}$ при $n \rightarrow \infty, n \in \Lambda$, для нормированного некоторым образом полинома $Q_{n}(z)$ справедлива следующая формула сильной асимптотики (ср. (2) и [8; (4.2)]): $Q_{n}(z)=\frac{\left(z-z_{n}\right)^{1 / 2}}{f(z)^{1 / 2}(A(z) V(z))^{1 / 4}} z^{(n+1) / 2} \exp \left\{n \psi(z) \pm \frac{1}{2} \Omega\left(z ; z_{n}\right)-u\left(z ; z_{n}\right)\right\}\left(1+O\left(\frac{1}{n}\right)\right)$.

Формула (8) справедлива равномерно на $K \backslash U_{\varepsilon}\left(z_{n}\right)$, где $K \subset D_{0}$ - произвольный компакт, $U_{\varepsilon}\left(z_{n}\right)$ - $\varepsilon$-окрестность $z_{n}$. Аналогичная формула имеет место и в $D_{\infty}$.

В типичном случае точки $z_{n}, n \rightarrow \infty$, всюду плотны на $\overline{\mathbb{C}}$, поэтому подпоследовательность с условием (7) существует, кроме того, ее плотность может быть сделана сколь угодно близкой к единице.

\section{Список литературы}

[1] А.И. Аптекарев, В.И. Буслаев, А. Мартинес-Финкельштейн, С.П. Суетин, УМН, 66:6(402) (2011), 37-122. [2] A. I. Aptekarev, M. L. Yattselev, arXiv: 1109.0332. [3] В. И. Буслаев, Матем. сб., 204:2 (2013), 39-72. [4] В.И. Буслаев, А. Мартинес-Финкельштейн, С.П. Суетин, Тр. МИАН, 279, МАИК, М., 2012, 31-58. [5] А. Мартинес-Финкельштейн, Е. А. Рахманов, С. П. Суетин, Матем. сб., 202:12 (2011), 113-136. [6] A. Martínez-Finkelshtein, E. A. Rakhmanov, S. P. Suetin, OPSFA '11, Contemp. Math., 578, Amer. Math. Soc., Providence, RI, 2012, 165-193. [7] А. Мартинес-Финкельштейн, Е. А. Рахманов, С. П. Суетин, УМН, 68:1(409) (2013), 197-198. [8] J. Nuttall, Constr. Approx., 2:1 (1986), 59-77. [9] H. Stahl, J. Approx. Theory, 91:2 (1997), 139-204. [10] С. П. Суетин, Матем. сб., 194:12 (2003), 63-92. [11] Г. Сегё, Ортогоналъные многочлены, Физматгиз, М., 1962, 500 с.

А. В. Комлов (А. V. Komlov)

Математический институт им. В. А. Стеклова РАН

E-mail: komlov@mi.ras.ru

\section{С. П. Суетин (S. P. Suetin)}

Математический институт им. В. А. Стеклова РАН

E-mail: suetin@mi.ras.ru
Представлено Д. В. Трещёвым Принято редколлегией 20.07.2013 